\title{
XXIII. On screens transparent only to ultra-violet light and their use in spectrum photography
}

\section{R.W. Wood}

To cite this article: R.W. Wood (1903) XXIII. On screens transparent only to ultra-violet light and their use in spectrum photography , Philosophical Magazine Series 6, 5:26, 257-263, DOI: 10.1080/14786440309462920

To link to this article: http://dx.doi.org/10.1080/14786440309462920

曲 Published online: 15 Apr 2009.

Submit your article to this journal $₫$

Џll Article views: 5

Q View related articles $\square$

Citing articles: 5 View citing articles ๘ 
coefficient of absorption equal to that of rock-salt and a thermal conductivity equal to that of cork. Curves I. and II. give the distribution of temperature when the average temperature is about $30^{\circ} \mathrm{C}$., Curve I. representing the steady state when the boundaries are totaliy reflecting, and Curve II. when they are black. Curve III. represents the steady state for totally reflecting boumdaries when the average temperature of the plate is about $360^{\circ} \mathrm{C}$. This curve shows the approach to the steady state, when the transmission of heat is entirely effected by radiation, thermal conductivity being neglected. The temperature distribution in the limiting case being uniform, but discontinuous at the surfaces. The numbers from which the curves are plotted are given in the following Table, which includes also under column IV. the case of black surfaces, with an average temperature of $360^{\circ}$. It is only necessary to give the temperatures near the colder bounding surface, as the distribution is symmetrical at both sides and uniform near the centre of the plate. The columns headed Temperature Differences represent the difference between the temperature at any point and the temperature of the colder surface; the complete temperature change in the plate is supposed to be $1^{\circ}$.

\begin{tabular}{|c|c|c|c|c|}
\hline \multirow{2}{*}{$\begin{array}{c}\text { Distance from } \\
\text { Colder } \\
\text { Boundary } \\
\text { in ems. }\end{array}$} & \multicolumn{4}{|c|}{ Temperature Differences. } \\
\hline & I. & II. & III. & IV \\
\hline$\cdot 01$ & $\cdot 075$ & 042 & 240 & 127 \\
\hline .02 & $\cdot 129$ & 074 & $\cdot 330$ & $\cdot 178$ \\
\hline$\cdot 04$ & $\cdot 196$ & $\cdot 117$ & 384 & .212 \\
\hline 0.5 & $\cdot 217$ & $\cdot 133$ & $\cdot 388$ & 221 \\
\hline 10 & 274 & $\cdot 186$ & 400 & .253 \\
\hline 90 & $33 \bar{o}$ & $\cdot 267$ & +25 & 315 \\
\hline
\end{tabular}

XXIII. On Screens T'ransparent only to Ultra-Violet Light and their Use in Spectrum Photography. By R. W. Wood, Professor of Experimental Physics, Johns Hopkins University*.

[Plates III. \& IV.]

AYONE who has repeated Tyndall's beautiful lecture A experiment of kindling a pine stick in the clark heat focus of a burning-glass, concentrating light from which the visible radiations have been removed by means of a solution

* Communicated by the Author.

Phil. Mag. S. 6. Vol. 5. No. 26. Feb. 1903. 
of iodine in bisulphide of carbon, must bave wished that we possessed a screen, opaque to visible light and transparent to the ultra-violet.

I have recently succeeded in making a screen quite transparent to these radiations, though a gas flame cannot be seen through it. By combining it with a large condensing-lens and an arc-lamp, it is possible to form a dark focus of ultraviolet light in which a lump of uranium nitrate glows with a vivid green phosphorescence like a great emerald.

Besides giving us the means of performing a most beautiful lecture experiment, these screens make it possible to photograph the ultra-violet lines in grating spectra of higher orders than the first, entirely uncontaminated by the visible radiations which overlie them. Other applications at once suggest themselves, such as the complete removal of the highly actinic blue and violet rays, in certain investigations of the ultra-violet region where the long exposures necessary are apt to produce fogging of the plates. It seems quite possible too, that photographs of the moon, planets, and nebulæ taken by means of ultra-violet light may furnish valuable data, as I shall attempt to show at the end of this paper.

The substance which has made possible the production of such a screen is nitroso-dimethyl-aniline, the remarkable optical properties of which I have already alluded to in a previous paper. As I have already said, a prism formed of this substance yields a spectrum about 30 times as long as a quartz prism of the same angle, the dispersion resembling somewhat that of selenium. I was of the opinion that the absorption, which commences at about wave-length $\cdot 0005$, would increase continuonsly from this point down to the end of the spectrum, as was found to be the case with selenium. On commencing a study of the absorption, however, I was astonished to find that it ended abruptly a little beyond the $H$ and $K$ lines, and that from this point on, the substance was transparent even down to the last cadmium line, of wavelength $\cdot 0002$.

It at once occurred to me that if some substance or substances could be found absorbing the red, yellow, and green, and transparent to the ultra-violet, we could, by combining them with the nitroso compound, produce the long-sought screen.

Very dense cobalt glass, coated with a thin film of gelatine lightly stained with the nitroso, was found to be transparent only to the extreme red and the ultra-violet, and the red was 
eventually removed by means of a thin sheet of Chance's "signal-green" glass, such as is used for one of the reflectors in the Ives Kromskop.

This combination is wholly opaque to visible light, while freely transmitting everything between wave-lengths 34 and 38 . Of course the employment of glass sereens limits the ultra-violet transmission, and a screen of this description is useful chiefly for lecture demonstrations. Considerable care must be used in the adjustment of the strength of the solution of the nitroso in gelatine, otherwise the intensity of the ultraviolet light is considerably weakened.

The best strength is such as will be just sufficient to remove the blue and violet light transmitted by dense cobalt glass. Quite a number of trials will be found necessary in adjusting the densities of the three components of the screen to secure the maximum effect, but when the balance is just right, it is possible to form a focus in which a piece of paper is quite invisible, while a mass of erystals of the nitrate of uranium (which I have found superior to anything else) glows with sufficient intensity to be seen from the back of the largest lecture-room.

It is best to exclude carefully all light which does not pass through the screen.

With the assistance of one of our students, I am at the present time investigating the absorption of a large number of substances, which, so far as I know, have not been previously studied, and $I$ hope in time to dispense with glass entirely, and produce an opaque screen which transmits ultriviolet down to the end of the spectrum.

A combination of a tube furnished with quartz ends, on one of which is a thin film stained with nitroso, transmits all of the ultra-violet, and only the extreme red, but it is very inconvenient to work with. For use as a screen in spectrumphotography there is no especial object in removing the reu, yellow, and green, the nitroso alone blocking out completely the actinic portions of the visible spectrum, which overlie the ultra-violet in the second and third order spectra, and I shall next consider solutions of the substance in various fluids in connexion with spectrum-photography.

I have found that the best method of quickly securing a record of the absorption of a solution is to bring a prismatic layer of the liquid, contained in a quartz cell, before the slit of a quartz spectrograph, and photograph the spectrum of the cadmium spark. We secure in this way a record of the absorption of the liquid in various thicknesses, in the form of 
a curve, quite similar to the curves laboriously constructed from the readings obtained with the spectrophotometer*

The curve obtained with a solution of the nitroso in glycerine is shown in Plate III. fig.. 4 and 5.

It will be noticed that ater a certain thickness has been passed we begin to get a noticeable absorption in the ultraviolet, the form of the curve in this region being well shown in figs. 3 and 4 . The band in the blue and violet is, however, so much heavier that, by employing a film of suitable thickness, we can get complete opacity in this region, combined with almost perfect transparency in the ultra-violet. The nitroso is soluble in water, glycerine, ether, alcohol, bisulphide of carbon, and many other Huids, and the region of heaviest absorption varies somewhat with the nature of the solvent, the shift of the band not, however, following Kundt's rule in every case.

A stained gelatine film on a quartz plate forms a fairly suitable screen, if we do not wisl to photograph below the group of cadmium lines at wave-length 2314. It is opaque, however, to wares much shorter than this. The glycerine solution transmits down to the last cadminm line, $\lambda=2147$, and some other solvents nppear to work equally well.

In photographing the spectrum of the cadmium-spark in the ultra-riolet of the second order, with the fourteen-foot concave grating, I found that the prolonged exposure of the solution in glycerine to the light of the spark resulted in its decomposition. Gas-bubbles formed in the thin quartz cell, and by bridging across the space between the two plates allowed the passage of blue and violet light.

The same thing occurred with pure glycerine under a quartz plate, while glycerine under glass was unaffected, showing that the decomposition was caused by the extreme ultra-violet.

In addition to the formation of bubbles, a gradual bleaching of the solution occurred. To obviate this difticulty I constructed

* It is my intention to prepare a monograph on the absorption of a large number of the aniline dyes, and other organic compounds such as the nitroso-dimethyl-aniline, which have not been previously investigated. The spectra will be approximately normal, all on the same scale, and will extend from the C-line down to the end of the spectrum. They will be photographed in the manner which I have described, and will, I hope, make it possible for the spectroscopist or physicist to pick out at once the combination necessary to produce any desired result. Preliminary experiments are now in progress to determine the best form to give the apparatus, and the most suitable source of light, and I shall be rery glad of any suggestions pertaining either to the apparatus or to particular substances worthy of investigation. [A similar method of studying absorption within the range of the visible spectrum was employed many years ago by the late Dr. Gladstone.-Ed. Phil. Mag.] 
a small cell of quartz, by cementing two plates together, with a space of about 0.5 millim. between them, the cell thus formed being cemented to the bottom of a small thistle tube with a very small bore. By filling the thistle tube with the glycerine solution a flow took place through the cell at the rate of abuut a drop every two minutes. This device worked admirably, and gave no trouble at all, the cell being placed close to the slit of the grating camera, in the path of the convergent beam from the quartz lens. Another very satisfactory screen can be made by dissolving cellnloid (previously boiled for some time in water) in amyl acetate, adding a little nitroso, and fowing the solution on a quartz plate. It is, however, opaque to the last two cadmium lines.

The use of the screen necessitates considerable increase in the time of the exposure, the amount varying from two to ten or even twenty times, according to the density of the screen.

The strength of the glycerine solution must be adjusted according to the work required of it: a strong solution gives a wider band in the blue and violet, but diminishes the intensity of the ultra-violet as well. In general the best results are obtained when the blue line of wave-length 4799 in the spark-spectrum of cadmium can be just barely discerned.

In Plate III. fig. 1 the wave-lengths of the principal lines in the spark-spectrum of cadmium are given for reference. The action of the nitroso screen is well shown in fig. 2, the spectra being photographed with a small quartz spectrograph made by Fuess. The first seven spectra were taken through the glycerine-nitroso cell which I have just described, with the following times of exposure: $5,10,15,20,30,45$, and 60 seconds. The cell was then removed, and the following six spectra taken with exposures $2 \frac{1}{2}, 5,10,15,20$, and 30 seconds. A siudy of these spectra enables ns to calculate just what can be done with this screen, and the mecessary increase in the time of exposure resulting from its use.

In fig 6, which is a negative, we have the absorption spectra of the various components of the screen which I mentioned in the first part of this paper, taken with exposures of 20 seconds each.

The spectra were taken through screens as follows:-

1. Nitroso in gelatine on glass (thin film).

2 . " " $"$ (thick film).

$3 . \quad " \quad " \quad$ on cobalt glass (thin film).

4. " " " ", (thick film) strong ultra-violet absorption.

5. Dense cobalt glass.

6. Turnbull's blue in gelatine. 
7. Chance's "Signal-green" glass (two thicknesses).

8. No screen, 3 seconds' exposure.

9. Cyanine in canada-balsam.

11. Aurantia in collodion.

12. Signal-green glass (one thickness).

These photographs were taken on an orthochromatic plate, the yellow and yellow-green being compressed into the small strip which appears alone by itself in No. 11.

The utility of the nitroso screen in photography with the concave grating is very clearly brought out in the photographs of the iron spectrum shown in Plate IV. These were mads with a 14-foot grating, with a glycerine nitroso cell before the slit during one of the exposures. Figures 1 and 2 are from the same plate. Strip B in each was made throngh the sereen, and shows the ultra-violet of the third order, uncontaminated by the blue of the second. In strips A, which were made without the screen, the two orders are mixed. Strips C were made through a glass screen, which cut off the third order ultra-violet, leaving the blue of the second. I have marked a few of the wave-lengths to aid in the identification of the lines.

The times of exposure were for strips $\mathrm{A}$ and $\mathrm{C}$ ten minutes, for B fifty minutes.

The group of cadmium lines in the neighbourhood of wave-length 2314 is, in the second order spectrum, mixed up with a lot of blue air lines of the first order spectrum. The separation of the two by the nitroso screen is well shown in fig. 3, in which the two orders are shown superposed in strips $A$, and the ultra-violet of the second order in strip B. The exposures in this case were 15 minutes and 2 hours respectively.

Another screen which I believe may prove useful in astrophysical work is made by combining nitroso-dimethylaniline with a small amount of the dye uranine, the latter removing the bluish-green portion of the spectrum which affects the photographic plate. By a proper adjustment of the two in gelatine on glass, a screen can be formed which, when used with an ordinary (i.e., not orthochromatic) plate gives us a photograph made exclusively by ultra-violet light, comprised between wave-lengths 345 and 365 , a rather narrow range.

I have made a few photographs with a screen of this description, which have brought out some interesting points. In a photograph of the full moon, taken by ultra-violet light, the contrast between the bright and dark areas is very strongly accentuated, while in photographs of landscapes made in the same way there is almost no contrast at all except between 
white objects and objects not white. I have also photographed a collection of rocks and minerals with ultra-violet light and with yellow light. In the negative taken by yellow light there is a great deal of contrast and detail, especially in the marbles and conglomerates, while in the negative taken by ultra-violet light all this is alsent, the white specimens coming out very black, with everything else of a thin and almost uniform grey. 1 hope in the near future to have an opportunity of making some lunar photographs on a large scale, the only instrument at my disposal at the present time being the nine-inch equatorial of the University. Photographing by ultra-violet light appears to diminish the contrast between all objects not white, and to increase the contrast between white objects and those not white. I do not wish to be hasty in drawing conclusions, but it appears to me to be probable that the more luminous portions of the lunar surface, if not as white as plaster-of-paris, must at least be much whiter than grey sandstone. In Plate IV. fig. 4 are reproduced two photographs of the same landscape, taken at the same time and under similar conditions of illumination, the one (A) taken on an orthochromatic plate by yellow light through a screen of dense aurantia, the other $(B)$ taken on an ordinary plate by ultra-vivlet light.

The absence of contrast between the chimneys and walls in $B$ is especially noticeable in the right-hand part of the picture. I tried various times of exposure, and the picture reproduced is the best of the lot. Another curious effect is the almost complete absence of shadows in the ultra-violet picture (it was taken in full sunlight like the other), showing that most of the ultra-violet light comes from the sky, which is what we should expeet, though we should hardly anticipate that the effect would be so pronounced. This is best seen on the monument and on the snow in the middle distance. The increase of "atmosphere" in the ultra-violet picture is very marked. It is so strong that under-exposed plates fog in the shadows of objects not over one hundred yards from the camera, a circumstance which shows the great scattering power of the air for these short waves. The two pictures are also interesting as showing that our eyes have developed a maximum sensibility for that region of the spectrum which shows terrestrial objects in strongest contrast. Nitrosodimethyl-aniline is the only substance, other than the ordinary aniline dyes, that I have examined thus. far, and I feel very hopeful of finding, among the large number of allied substances, absorbing media even more transparent to the ultraviolet radiations than the one which I have described in this paper. 
Phil. Mag. S. 6. Vol. 5. Pl. III.

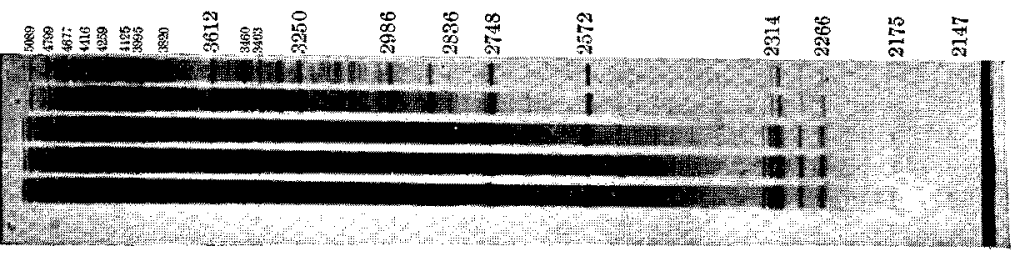

Fis. 1.

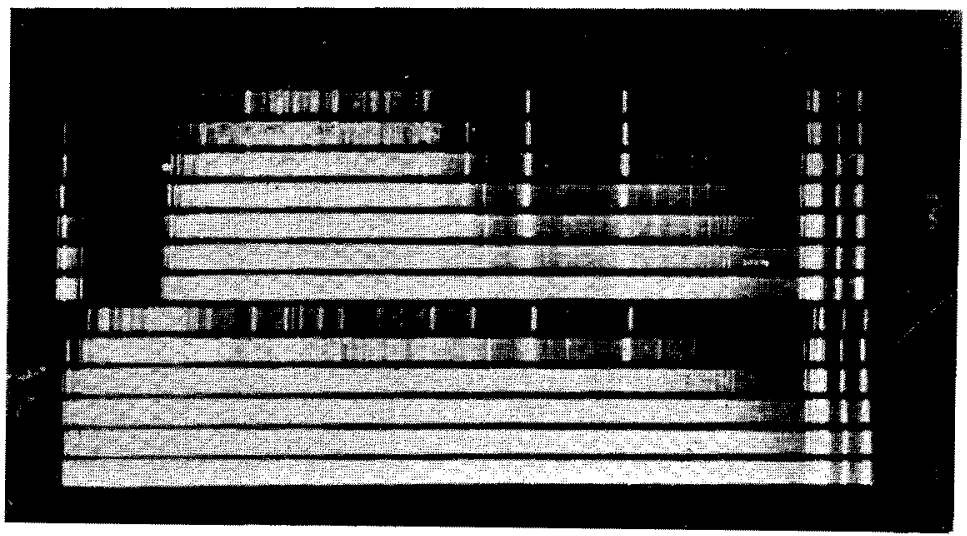

Fig. 2 .

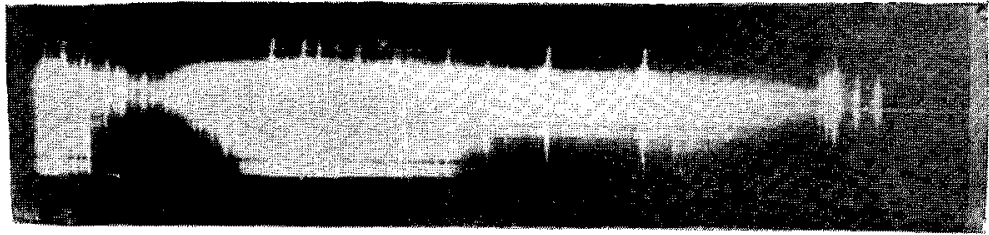

Fix. 3.

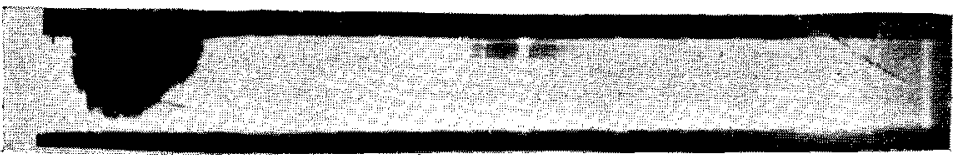

Fin. 4.

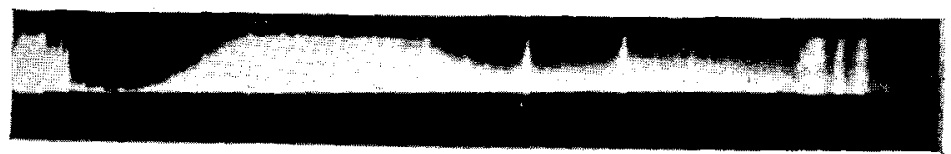

Fia. 5.

$5 \% 8$,

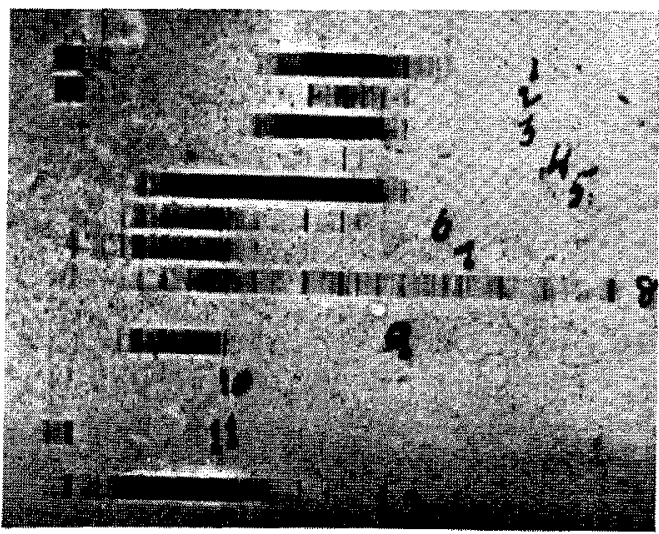


Phil. Mag. S. 6. Vol. 5. Pl. IV.

Fig. 1.

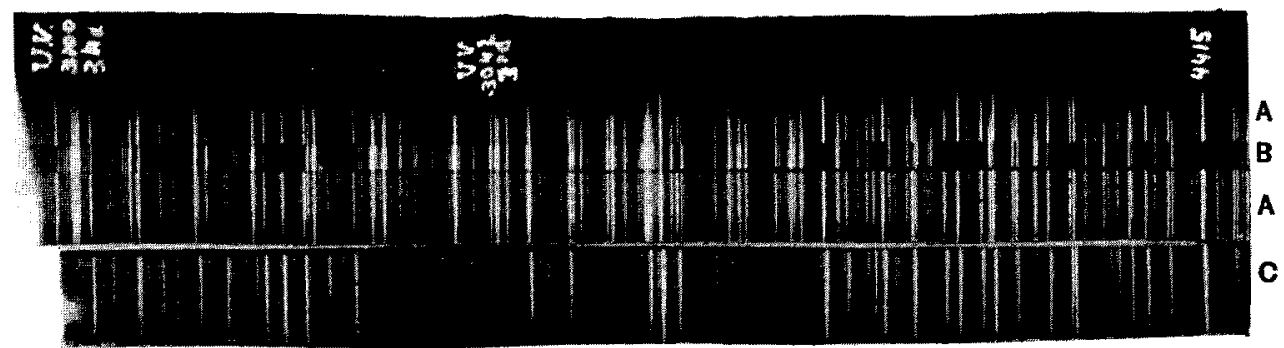

Fig. ?.

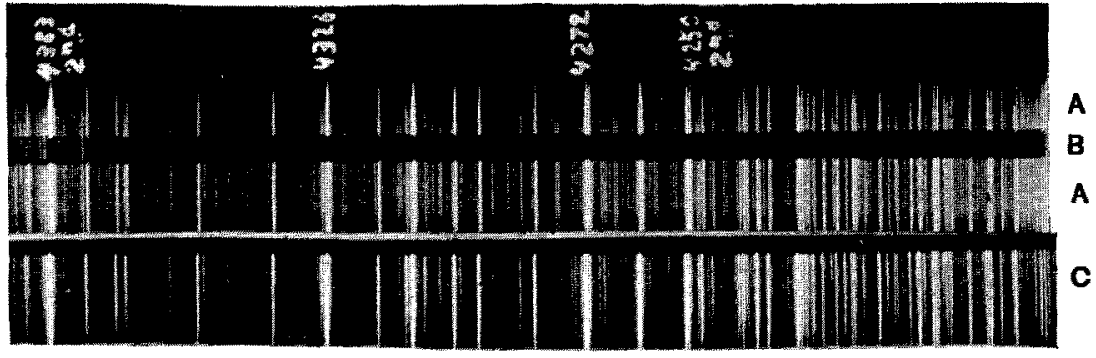

Fig. 3 .

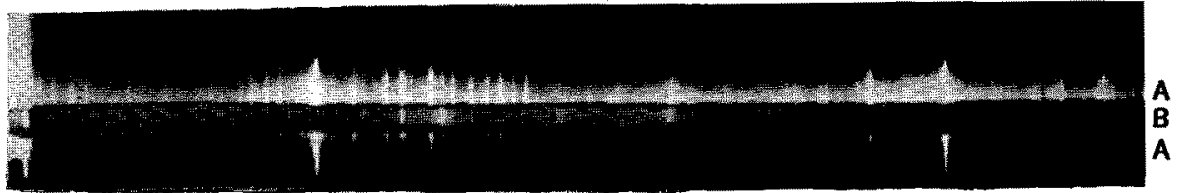

Fig* 1.

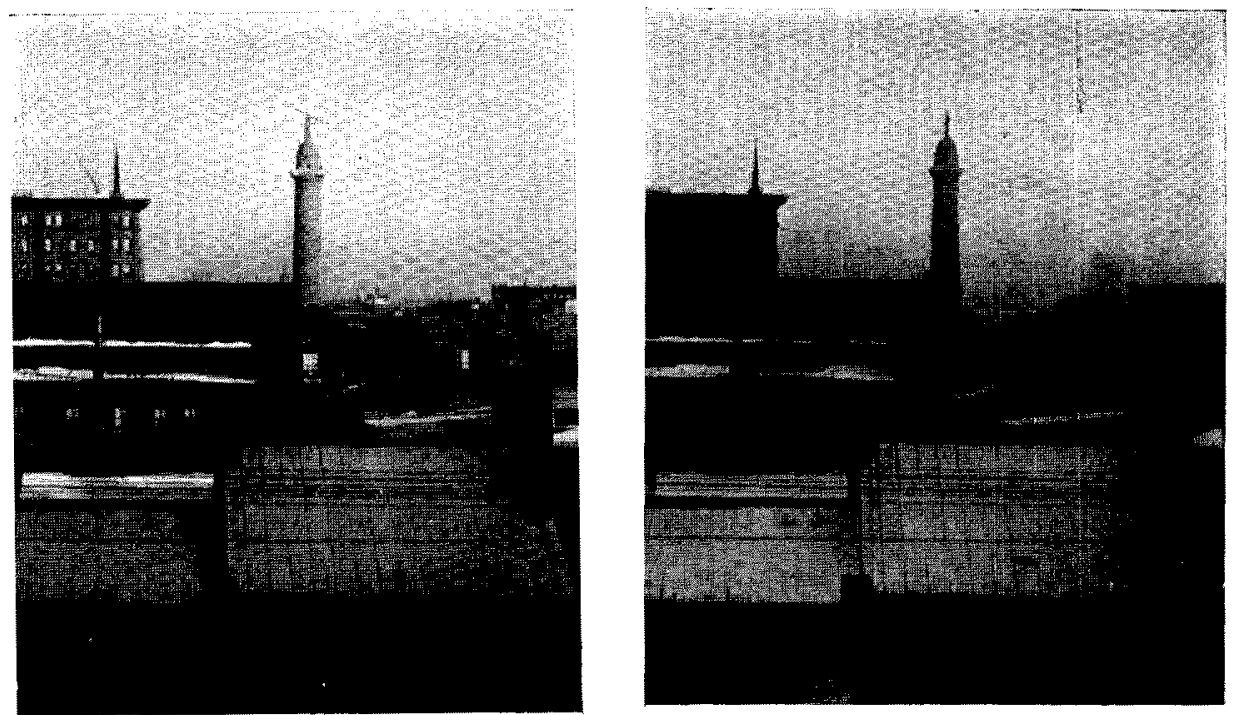

B. 